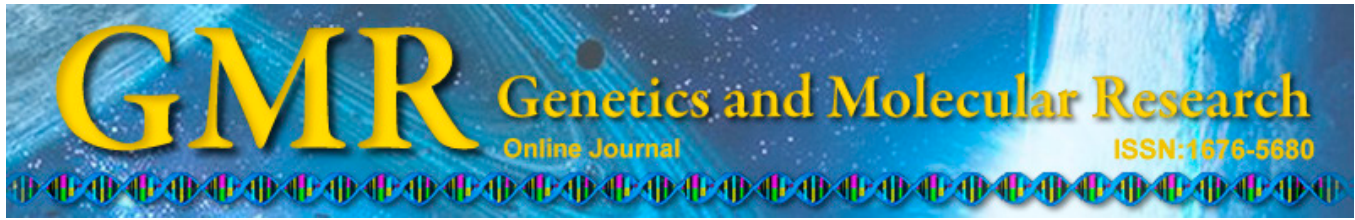

\title{
Molecular cloning and expression analysis of female sterile homeotic gene $(f s h)$ in the oriental river prawn Macrobrachium nipponense
} W.Y. Zhang ${ }^{1,2}$, S.F. Jiang ${ }^{2}$, Y.W. Xiong ${ }^{2}$, H.T. Fu ${ }^{1,2}$, H. Qiao
, S.M. Sun

${ }^{1}$ Wuxi Fisheries College, Nanjing Agricultural University, Wuxi, China ${ }^{2}$ Key Laboratory of Freshwater Fisheries and Germplasm Resources Utilization, Ministry of Agriculture, Freshwater Fisheries Research Center,

Chinese Academy of Fishery Sciences, Wuxi, China

Corresponding author: H.T. Fu

E-mail: fuht@ffrc.cn

Genet. Mol. Res. 14 (2): 4318-4330 (2015)

Received May 6, 2014

Accepted October 6, 2014

Published April 30, 2015

DOI http://dx.doi.org/10.4238/2015.April.30.4

ABSTRACT. The gene female sterile homeotic ( $f$ sh) plays crucial roles in molecular function, including protein kinase activity and DNA binding, which are involved in biological processes such as terminal region determination and negative regulation of DNA-dependent transcription. Although $f s h$ has been found in Drosophila melanogaster, little is known regarding its expression in crustaceans. In this study, a $f_{s} h$ gene homologue, designated as $M n f s h$, was cloned and characterized from the testis of the oriental river prawn, Macrobrachium nipponense, by using EST analysis and the RACE approach for the first time. The full-length cDNA of Mnfsh was $2029 \mathrm{bp}$, consisting of a 5' UTR of 361 bp, a 3' UTR of $216 \mathrm{bp}$, and an ORF of $1452 \mathrm{bp}$ encoding 484 amino acids. qRT-PCR analysis showed that the $M n f s h$ gene was expressed in the testis, ovary, muscle, heart, eyestalk, and abdominal ganglion, with 
the highest level of expression in the ovary and the lowest in the heart. qRT-PCR analyses showed that the expression levels of Mnfsh mRNA both significantly increased in the zoea stage, the VII larvae, and 1st day post-larvae after metamorphosis. In conclusion, the results of the present study indicate that Mnfsh is an arthropod $f s h$ homologue and probably also plays important roles in embryogenesis, organogenesis, and morphological differentiation of $M$. nipponense.

Key words: Female sterile homeotic ( $f$ sh); Macrobrachium nipponense; Gene cloning; Gene expression

\section{INTRODUCTION}

More than a dozen trithorax group $(\operatorname{tr} x G)$ proteins are involved in activation of Drosophila HOX genes. The maternal-effect gene female sterile (1) homeotic $\left[f_{s}(1) h\right.$, also called $f s h$ ] is one of the $\operatorname{trx} G$ genes (Gans et al., 1975) and was identified as the most important transactivator of Ultrabithorax ( $U b x)$ activation by its strong genetic interactions with Ubx, trithorax (trx), and absent, small or homeotic discs 1 (ash1) mutations (Digan et al., 1986; Shearn, 1989). Additionally, it has been reported to be related to female reproduction and growth in Drosophila melanogaster (Gans et al., 1980; Haynes et al., 1989). Because Ubx expression impacts where the thoracic segments and abdominal segments connect in the Drosophila embryo, lack of functional $f s h$ is known to cause complex developmental defects including homeotic transformation and early embryonic lethality (Forquignon, 1981; Huang and Dawid, 1990). Previous studies showed that zygotic mutations cause either lethality or female sterility, whereas maternal mutations cause segmental deletions and thoracic homeotic transformations (Rhee et al., 1998; Florence and Faller, 2008). Considering that crustaceans have a dramatically close evolutionary relationship with insects (Budd and Telford, 2009; Zhang et al., 2012), we hypothesize that $M n f s h$ is involved in the regulation of embryonic development in crustaceans.

$f s h$ recognition proteins have been isolated and characterized in other species such as Drosophila melanogaster (Haynes et al., 1989), Camponotus floridanus, and Harpegnathos saltator (Bonasio et al., 2010). However, little information is available to date on temporal expression of $f s h$ mRNA during embryonic and larval development in crustaceans. The oriental river prawn, Macrobrachium nipponense, (Crustacea; Decapoda; Palaemonidae) is a commercial freshwater prawn species that is widely distributed in many Asian countries including China, Japan, Korea, Vietnam, and Myanmar (Hongtuo et al., 2012; Qiao et al., 2013). This species is considered an important fishery resource in China, with an annual production of 230,248 tons (Bureau of Fishery, Ministry of Agriculture, and P.R.C., 2011). In recent years, our laboratory has performed some molecular research on the M. nipponense development system (Zhang et al., 2012, 2013a,b, 2014). To further understand the molecular mechanism of maturation, other gene regulations involved in the developmental process also need to be examined in M. nipponense. In this study, we cloned a full-length $f s h$ cDNA from oriental river prawn and analyzed its expression pattern throughout developmental stages, which could help improve our understanding of the regulatory mechanism of early embryonic and post-embryonic development in the oriental river prawn. 


\section{MATERIAL AND METHODS}

\section{Animal and sample preparation}

Sexually mature adult oriental river prawns, weighing about 2.0-5.6 g, were obtained from Tai Lake $\left(120^{\circ} 13^{\prime} 44^{\prime \prime E}, 31^{\circ} 28^{\prime} 22^{\prime \prime} \mathrm{N}\right)$ in Jiangsu Province, China from February to May 2012 and were reared in aerated freshwater for $72 \mathrm{~h}$ at $28^{\circ} \mathrm{C}$ before tissues were collected. For cDNA synthesis and tissue distribution pattern, the testis, ovary, muscle, heart, eyestalk, and abdominal ganglion were dissected from healthy mature individuals. Different developmental stages of embryos, larvae, and post-larvae were obtained from our laboratory. Each developmental stage of embryos was determined and collected following the criteria by Chen et al. (2012); the embryos were then observed and pictures were taken with a fluorescence microscope (OLYMPUS BX61, Japan). Larvae were collected every 4 days between 1 day post-hatching (LI) and LVIII (1 day before metamorphosis). Post-larvae were collected every 5 days between 1 and 20 days after metamorphosis (PL1-PL20) and every 10 days between PL20 and PL30. All samples were washed with 1X phosphate-buffered saline $(0.01 \mathrm{M})$, frozen immediately in liquid nitrogen, and stored at $-80^{\circ} \mathrm{C}$ until RNA extraction.

\section{Isolation of total RNA and for real-time quantitative polymerase chain reaction}

Total RNA was extracted from the testis of adult prawns using RNAiso Plus Reagent (TaKaRa, Japan) according to manufacturer protocols. The isolated RNA was treated with RNase-free DNase I (Sangon, China) to eliminate possible genomic DNA contamination. The concentration of each total RNA sample was then measured by BioPhotometer (Eppendorf, Germany), and $2 \mu \mathrm{L}$ was analyzed on a $1 \%$ agarose gel to check the integrity. The cDNA was synthesized from $5 \mu \mathrm{g}$ of total RNA by the PrimeScript ${ }^{\mathrm{TM}}$ RT-PCR kit (TaKaRa, Japan). Reaction conditions used were recommended by the manufacturer. The cDNA was kept at $-20^{\circ} \mathrm{C}$ for real-time quantitative polymerase chain reaction (qRT-PCR).

\section{Sample analysis and calculation}

A gene-specific primer set was designed based on expressed sequence tags (EST) sequences (ALB High Quality_EST.seq.Contig868) of $f$ sh homologue obtained from the M. nipponense testis cDNA library (Qiao et al., 2012). The cDNA ends of the $f$ sh gene was isolated through rapid-amplification of cDNA ends (RACE) PCR with the 5'-full RACE kit (TaKaRa) and 3'-full RACE kit (TaKaRa) using a nested PCR strategy using a thermal cycler (Eppendorf) according to manufacturer protocols (Amparyup et al., 2008). The total RNA of the testis tissues was used as a template to amplify the cDNA ends. Gene-specific primers of $M n f s h$ were designed based on the known fragment. All primers used in this study are shown in Table 1.

The PCR products were gel-purified and ligated into the pMD18-T vector (TaKaRa) following manufacturer instructions. The recombined products were then transformed into Escherichia coli DH5a-competent cells (Qiagen, Germany), which were identified by blue/ white screening and confirmed by PCR. Three of the positive clones were sequenced in both directions using an automatic DNA sequencer (ABI Applied Biosystems Model 3730), and these resulting sequences were verified and subjected to cluster analysis in National Center for Biotechnology Information (NCBI). 
Table 1. Sequences of primers employed in this study for the $f$ sh gene of Macrobrachium nipponense.

\begin{tabular}{|c|c|}
\hline Primer & Primer sequence $\left(5^{\prime}-3^{\prime}\right)$ \\
\hline \multicolumn{2}{|c|}{ Primers for the middle of the sequence } \\
\hline$f_{s} h$ middle 5 ' primer & ATCACACACCAACGCCCGTC \\
\hline$f_{s h}$ middle $3^{\prime}$ primer & GTGAGTGGCTGTTAGCGGCG \\
\hline \multicolumn{2}{|l|}{ Primers for 3' RACE PCR } \\
\hline$f_{s h} 3^{\prime}$ out primer & TGCTCCTACTCCGTCTCCA \\
\hline$f s h 3^{\prime}$ in primer & CTTTCCTCGCCGCTAACA \\
\hline 3' RACE Outer primer & TACCGTCGTTCCACTAGTGATTT \\
\hline 3' RACE Inner primer & CGCGGATCCTCCACTAGTGATTTCACTATAGG \\
\hline \multicolumn{2}{|l|}{ Primers for 5' RACE PCR } \\
\hline$f s h 5^{\prime}$ out primer & AAATTCAACTTGACCGCATC \\
\hline$f_{s h} 5^{\prime}$ in primer & GTGATGGAAGGAATGGTG \\
\hline 5' RACE Outer primer & CATGGCTACATGCTGACAGCCTA \\
\hline 5' RACE Inner primer & CGCGGATCCACAGCCTACTGATGATCAGTCGATG \\
\hline \multicolumn{2}{|c|}{ Primers for qRT-PCR analysis } \\
\hline Mnfsh 5' primer & GTGACCAGGCTCCGCCAATCAG \\
\hline Mnfsh 3' primer & TGACGGGCGTTGGTGTGTGATG \\
\hline$\beta$-actin $5^{\prime}$ primer & TATGCACTTCCTCATGCCATC \\
\hline$\beta$-actin 3' primer & AGGAGGCGGCAGTGGTCAT \\
\hline
\end{tabular}

\section{Nucleotide sequence and bioinformatic analyses}

Searches for protein sequence similarities were conducted with NCBI's basic local alignment search tool (BLAST) algorithm (http://www.ncbi.nlm.nih.gov/BLAST/). The protein prediction was performed using the ORF Finder (http://www.ncbi.nlm.nih.gov/gorf/). The ProtParam program (http://www.expasy.ch/tools/protparam.html) was used to compute physical and chemical parameters of the amino acid sequence. The motif was performed with the Motif Scan program (http://hits.isb-sib.ch/cgi-bin/motif_scan/). Multiple alignments of $f s h$ were generated using the DNAMAN program (http://www.lynnon.com/). A phylogenetic tree was constructed using the neighbor-joining (NJ) algorithm with 1000 bootstraps (http://www. megasoftware.net/; Tamura et al., 2011).

\section{Temporal and spatial expression patterns analyzed by qRT-PCR}

The Mnfsh mRNA expressions at different stages from embryo to post-larva and various adult tissues were measured by a SYBR Green qRT-PCR in a CFX96TM Real-Time System (Bio-Rad, USA). Gene-specific primers (Table 1 ) were used to amplify the $f s h$ gene, and the PCR products were sequenced to verify the specificity of the PCR primers. The $\beta$-actin primers (Table 1) were used to amplify the $\beta$-actin fragments that were used as an internal control (Zhang et al., 2010). Amplifications were performed on a 96-well plate with a $20 \mu \mathrm{L}$ reaction volume containing $10 \mu \mathrm{L}$ of $2 X$ SYBR Green Premix Ex Taq (TaKaRa), $0.2 \mu \mathrm{L}$ of each primers $(10 \mu \mathrm{M}), 2 \mu \mathrm{L}$ of template, and $7.6 \mu \mathrm{L}$ of PCR-grade water. The PCR temperature profile was $95^{\circ} \mathrm{C}$ for $30 \mathrm{~s}$ followed by 40 cycles of $94^{\circ} \mathrm{C}$ for $15 \mathrm{~s}, 60^{\circ} \mathrm{C}$ for $20 \mathrm{~s}$, and $72^{\circ} \mathrm{C}$ for $20 \mathrm{~s}$, with a $0.5^{\circ} \mathrm{C} / 5 \mathrm{~s}$ incremental increase from $60^{\circ} \mathrm{C}$ to $95^{\circ} \mathrm{C}$. Each sample was run in triplicate along with the internal control gene. To ensure that only one PCR product was amplified and detected, the dissociation curve analysis of amplification products was performed at the end of each PCR. The relative copy number of Mnfsh mRNA was calculated according to the $2^{-\Delta \Delta \mathrm{CT}}$ comparative cycle threshold (CT) method (Livak and Schmittgen, 2001).

\section{Statistical analysis}

All data are reported as means $\pm \mathrm{SD}$ (standard deviation) $(\mathrm{N}=3)$. Statistical analysis 
was performed using the SPSS software 19.0. Statistical significance was determined using one-way ANOVA and Tukey's multiple range tests. Significance was set at $\mathrm{P}<0.05$.

\section{RESULTS}

\section{cDNA cloning and sequence characterization of $f s h$}

The full-length cDNA sequence of $M n f s h$ was determined by merging the sequences of the $3^{\prime}$ and 5' RACE products. The coding nucleotide sequence and corresponding predicted amino acid sequences were analyzed, and the $M n f s h$ nucleotide and deduced amino acid sequences are shown in Figure 1. The Mnfsh cDNA was comprised of 2029 bp, containing $361 \mathrm{bp}$ in the 5'-untranslated region, $1452 \mathrm{bp}$ in the open reading frame (ORF), and $216 \mathrm{bp}$ in the $3^{\prime}$-untranslated region with two potential polyadenylation sites (AATAAA) located upstream of the poly(A) tail. The deduced Mnfsh contains 483 amino acid residues and shows $51 \%$ identity with the female sterile homeotic protein of Harpegnathos saltator (GenBank Accession: EFN88794.1) and about 50\% identity with that of Camponotus floridanus (GenBank Accession: EFN69688.1). Analysis of the deduced protein sequence of the Mnfsh showed that it was comprised of 483 amino acids with a predicted molecular mass of $52.641 \mathrm{kD}$ and an isoelectric point of 8.78. Conserved sequence and characteristic motifs of two bromodomains were identified in the deduced amino acid sequences of $M n f s h$. The sequence data have been deposited in the GenBank DNA database under the accession number KC517379.

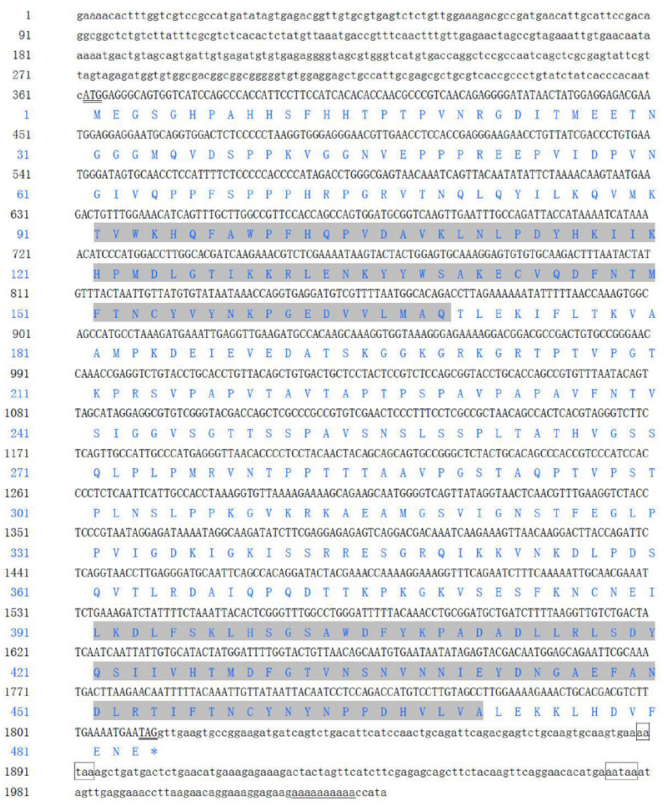

Figure 1. Mnfsh nucleotide (above) and deduced amino acid (below) sequences. The 3' UTR and 5' UTR are shown by lowercase letters. ORFs are shown by capital letters; the start (ATG) and stop (TAG) codons are double underlined, polyadenylation signal (AATAA) is in the boxes, polyadenylation is represented by a single underline, and the two putative bromodomains are shaded. 


\section{Similarity comparison and phylogenetic tree analysis}

The sequence alignment was used to determine the percent identity and similarity of amino acid residues with other $f s h$ genes. Sequence comparisons of the $M n f s h$-deduced amino acids showed identity of 51, 50, and 74\% to the female sterile homeotic protein of Harpegnathos saltator (Accession No. EFN88794.1), Camponotus floridanus (Accession No. EFN69688.1), and D. melanogaster (Accession No. NP_727228.1), respectively. Multiple alignments revealed that Mnfsh displayed a high degree of identity with $f s h$ of other arthropods (Figure 2). Therefore, we deduce that the obtained $M n f s h$ sequence from the foregoing analysis was the $f s h$ of $M$. nipponense.

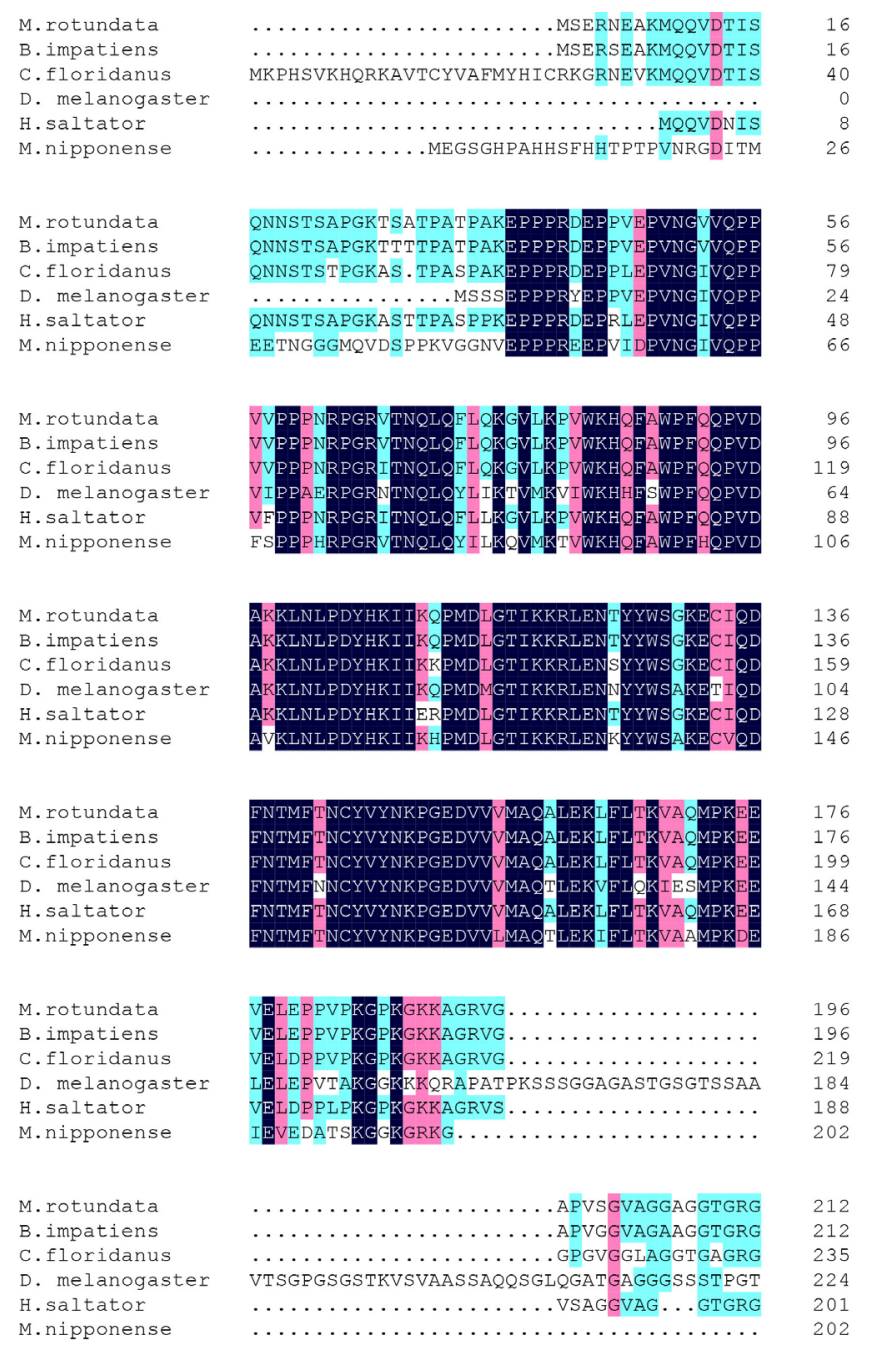

Figure 2. Alignment of $M n f s h$ amino acid sequences with other species using DNAMAN. Species names are abbreviated at the left and represent the following sequences obtained from GenBank: M. rotundata (XP 003705173.1), B. impatiens (XP_003490841.1), C. floridanus (EFN69688.1), D. melanogaster isoform A (NP_727228.1), H. saltator (EFN88794.1), and M. nipponense (in this study, KC517379).

Continued on next page 
Figure 2. Continued.

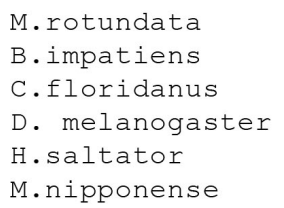

M.rotundata

B.impatiens

C.floridanus

D. melanogaster

H.saltator

M.nipponense

M.rotundata

B.impatiens

C.floridanus

D. melanogaster

H.saltator

M.nipponense

M.rotundata

B.impatiens

C.floridanus

D. melanogaster

H.saltator

M.nipponense

M.rotundata

B.impatiens

C.floridanus

D. melanogaster

H.saltator

M.nipponense

M.rotundata

B.impatiens

C.floridanus

D. melanogaster

H.saltator

M.nipponense

M.rotundata

B.impatiens

C.floridanus

D. melanogaster

H.saltator

M.nipponense

M.rotundata

B.impatiens

C.floridanus

D. melanogaster

H. saltator

M.nipponense

$\begin{array}{lll}\text { RPSSVAVAVTSSVPNSLTPSATSAGTTGVIPMPPLGTQAP } & 252 \\ \text { RPSSGAAAVTSSVNSLTPSATSAGTTGVIPMPPLGTQAP } & 252 \\ \text { RPASGAAAVTSSVPNLTPSATSAGTTGVIPMPPLGTQAP } & 275 \\ \text { QPGSGAGGAIAARPVSAMGGTVSSTAGGAPSIPPISTMPP } & 264 \\ \text { RPASGAAAVTSSVPNSLAPSATAARTTGVIPMPPLGTQAP } & 241 \\ \text {.........RTPTVPGTKPRSVAPVTAVTAPTPSPAVPAP } & 234\end{array}$

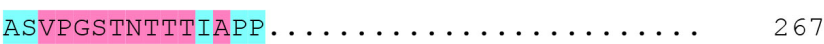

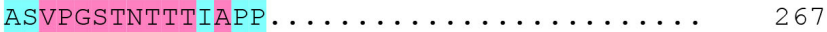

VSVPGSTNTTTIAPP.............. 290

HTVPGSTNTTTTAMAGGVGGPGAAGANPNAAALMASLLNA 304

VSVPGSTNTTTIAPP.............. 256

AVFNTVSIGGVSGTT................. 249

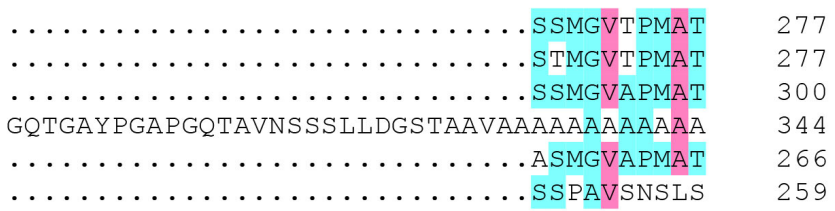

HNSLPQQVVPPTSGYHAQPAMDPQAASAVPPPPQVPTAPT 317 HNSLPQQVVP PT SGYHAQPAMDPQAASAVP P P PQVPTAPT 317 HNSLPQQVVPPTTGYHAQPAMDPQAASAVP P PPQVPTTPT 340 AAGGAAGAAGGAGTI PAVAVNAANAVQAYVNAGVSVGVDA 384 HNSLPQQVVPPTTGYHAQPAMDPQAASAVLPPPQVPTAPA 306 SPLTATHVGSSQLPLPMRVNTPPTTTAAVPGSTAQPTVPS 299

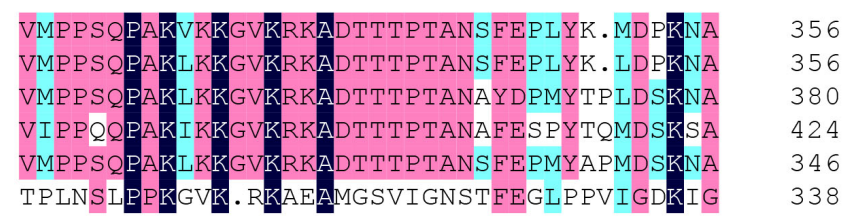

$\begin{array}{ll}\text { KIPARRESGRQIKKPTRQAEDGLVPFHQANMPLIGAMAQQ } & 396 \\ \text { KIPTRRESGRQIKKPTRQAEDGLVPFHQANMPLIGAMAQQ } & 396 \\ \text { KIPMRRESGRQIKKPTRQAEDGLVPYHQANMPLMGAMAQQ } & 420 \\ \text { KIATRRESNRQDLTFQGSGYNMS PLGVSGVPGLGGLVAGG } & 464 \\ \text { KIPTRRESGRQIKKPTRQAEDGLVPFHQTNMPLMGAMAQQ } & 386 \\ \text { KISSRRESGRQIKKVNKDLPDSQVTLRDAIQPQD...... } & 372\end{array}$

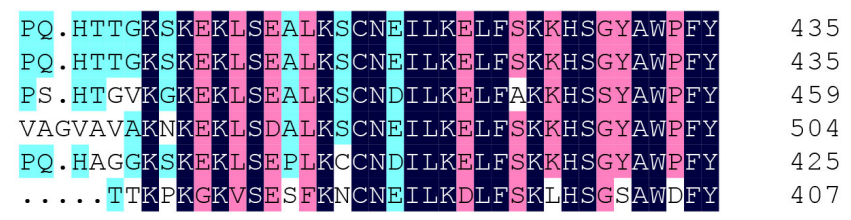

Continued on next page 
Figure 2. Continued.

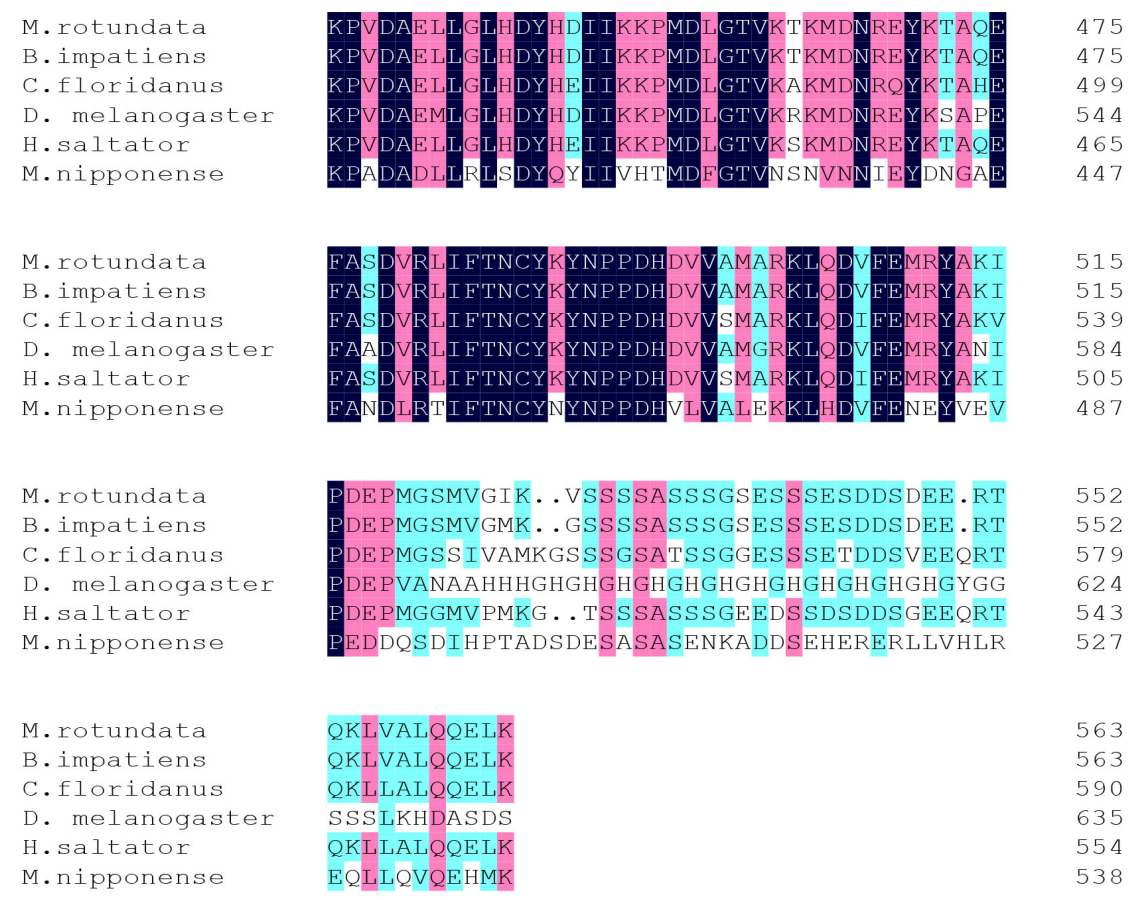

A condensed phylogenetic tree was constructed with MEGA 5.0 based on the NJ method using the complete $f s h$ sequence deposited in NCBI in order to study the relationship between $M n f s h$ and other well-defined $f_{s} h$ of arthropods. The NJ tree showed that the Mnfsh was within the crustacean clade (Figure 3), which is consistent with the evolution of species.

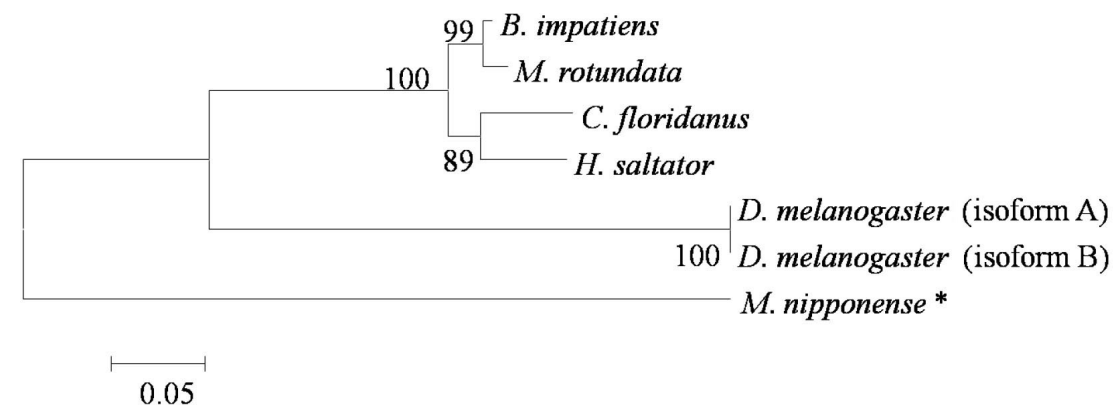

Figure 3. The neighbor-joining phylogenetic tree based on the sequences of $f$ sh from different species with MEGA 5.0. Species names and types of $f s h$ are listed on the right of the tree. Accession numbers in GenBank as follows: M. nipponense (in this study, KC517379), B. impatiens (XP_003490841.1), M. rotundata (XP_003705173.1), C. floridanus (EFN69688.1), H. saltator (EFN88794.1), D. melanogaster isoform A (NP_727228.1), and D. melanogaster isoform B (AAF46312.3). 


\section{Gene expression of $f s h$ in different tissues and stages}

Knowing that $\beta$-actin acts as an internal control in all tissues, qRT-PCR was employed to investigate the distribution of Mnfsh in different tissues. Mnfsh was expressed in all examined tissues of adult prawns, but at varying levels. Prominent expression was observed in the ovary (6.87-fold), followed by the eyestalk (4.91-fold), muscle (3.94-fold), and testis (2.83fold) at moderate levels; the lowest expression was in the abdominal ganglion (1.11-fold) and heart (the calibrator) (Figure 4).

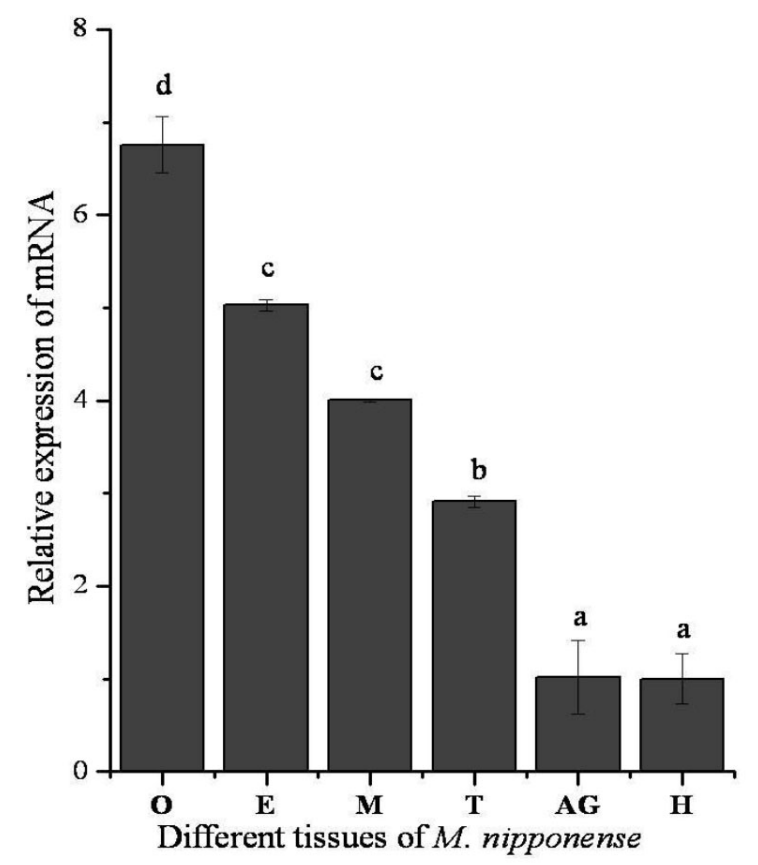

Figure 4. Relative expression levels of $M n f s h$ transcripts quantified in various adult tissues. Bars represent the triplicate mean $\pm \mathrm{SD}(\mathrm{N}=3)$. Bars with different letters differed significantly $(\mathrm{P}<0.05)$. O: ovary, E: eyestalk, $\mathrm{M}$ : muscle, $\mathrm{T}$ : testis, AG: abdominal ganglion, $\mathrm{H}$ : heart.

qRT-PCR results showed that the $M n f s h$ expression level significantly changed during the embryonic, larval, and post-larval stages (Figure 5). The temporal profile ranged from a peak of high expression to a minimun of moderate expression. Peak expression was observed in the mature ovum $(\mathrm{O})$ and higher than that at the first embryonic stage (cleavage stage, CS). Then, it sharply dropped by $98.24 \%$ at the blastula stage (BS). Subsequently, it remained stable until the nauplius stage (NS). As the embryo developed, the expression level of Mnfsh remarkably increased from the NS to zoea stage (ZS). In the larvae, the expression level increased roughly and reached the highest at the LVII. However, it abruptly decreased in late larvae (LVIII, just before metamorphosis). Larvae underwent metamorphosis, at which time the larvae transition into post-larvae resemble miniature adults. During the post-larvae stage, the highest expression of Mnfsh was found at the PL1, and it quickly decreased with progression of the post-larvae stage, but maintained low level after PL10. 


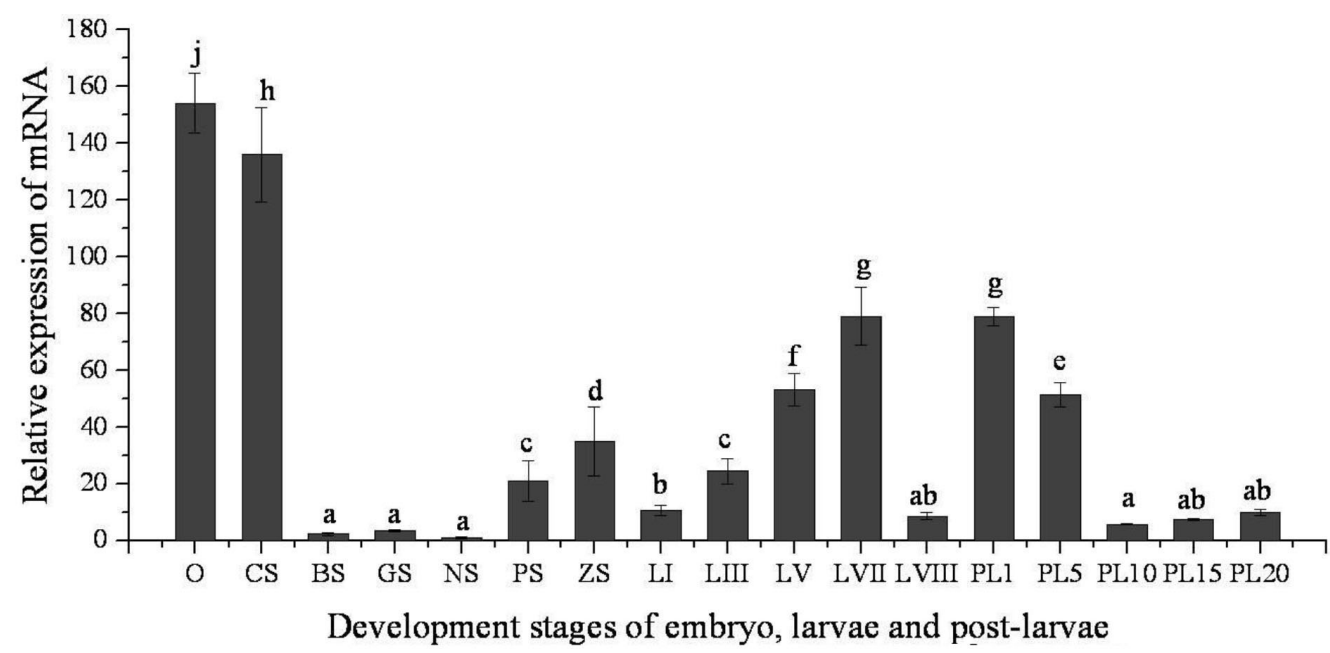

Figure 5. Mnfsh expression, normalized to $\beta$-actin, quantified in Macrobrachium nipponense in the ovum, embryos, and larvae before metamorphosis and post-larvae after metamorphosis. Bars represent the triplicate mean $\pm \mathrm{SD}(\mathrm{N}=$ 3). Bars with different letters significantly differed $(\mathrm{P}<0.05)$. O: ovum, $\mathrm{CS}$ : cleavage stage, BS: blastula stage, GS: gastrula stage, NS: nauplius stage, PS: protozoea stage, ZS: zoea stage. LI: first larva, PL1: first day post-larva after metamorphosis, PL5: fifth day post-larva after metamorphosis, PL10: tenth day post-larva after metamorphosis, PL15: fifteenth day post-larva after metamorphosis, PL20: twentieth day post-larva after metamorphosis.

\section{DISCUSSION}

In the present study, the complete cDNA sequence of $f_{s} h$ was cloned from the $M$. nipponense and verified by the homology PCR approach and RACE techniques. Conserved sequence and characteristic motifs of bromodomains were identified in the deduced amino acid sequences of $M n f s h$. Compared with other invertebrate nucleotide sequences (Haynes et al., 1992; Tamkun et al., 1992; Thorpe et al., 1996), the highest degree of sequence similarity was found near the two internal bromodomain repeats. The bromodomain, a motif initially identified in the Drosophila gene brahma (Tamkun, 1995) that is an extensive family of evolutionarily conserved protein modules originally found in proteins associated with chromatin and in nearly all nuclear histone acetyltransferases, has been discovered to function as an acetyl-lysine binding domain (Zeng and Zhou, 2002). The bromodomain consists of a 60-110 amino acid residue motif that has the potential to form two helices (Haynes et al., 1992; Marmorstein and Berger, 2001); it is conserved in eukaryotes and is commonly found in transcriptional regulatory proteins with diverse transcription-related functions (Jeanmougin et al., 1997). An additional homology among the $f s h$ sub-class has been observed at the extreme C-termini of the proteins and designated as the extra terminal (ET) domain (Lygerou et al., 1994). The functions are not yet known for the ET domain. Our study cloned the full-length cDNA of the Mnfsh gene, which encodes a protein with two bromodomains, but the ET domain was not observed. A potential explanation is that the evolution between species led to the lack of ET domain in Mnfsh.

In the present study, the transcripts of $f$ sh in $M$. nipponense were detected in all examined tissues; previous studies also found similar results (Chintapalli et al., 2007; Florence and Faller, 2008). The expression level of $M n f s h$ mRNA in the ovary was significantly higher than that in the testis, and it was explained that $f s h$ is a maternal-effect gene. Interestingly, peak 
expression observed also in the ovary, followed by eyestalk, indicating that the effective functioning of the eyestalk, which is a secretory organ, in oriental river prawn could be affected by $f s h$ when necessary.

In the present study, Mnfsh $m R N A s$ were expressed at every stage including the cleavage stage during the embryonic development of $M$. nipponense; this result is similar to those of previous studies that revealed that the gene plays an important role in development and reproduction. In particular, the mutations result in sterility and homeotic transformations (Gans et al., 1975, 1980; Digan et al., 1986), and its direct role in homeotic gene activation has been ascertained by complex phenotypes in mutant embryos in Drosophila (Huang and Dawid, 1990). The embryonic developmental process of M. nipponense can generally be divided into six stages: CS, BS, GS, NS, PS, and ZS. Our results found that the $\mathrm{O}$ had the greatest expression of $M n f s h$, which indicates that Mnfsh mRNA may have been maternally provided in the ovum, which provides strong evidence that it is a maternal-effect gene. After fertilization, Mnfsh gene expression began to decline from the transition of cells from gametes to zygotes. CS expression was the highest in the embryonic developmental process, and then sharply decreased in the BS, retaining a low level in the NS. Furthermore, the relative expression of mRNA during the NS to ZS rose to $30 \%$ of the maximum, when organogenesis was most active, which was consistent with a previous finding that the organogenesis in the embryos started at the NS (Zhang et al., 2010). Therefore, our results demonstrated that Mnfsh is closely related to histo-differentiation and organogenesis during the embryonic developmental process. After ZS, M. nipponense ruptured out from embryonic membranes in 2 days and molted. In the larvae, the expression increased roughly and reached the highest level at the LVII stage; however, it abruptly decreased in the late larvae (LVIII, just before metamorphosis). After metamorphosis, the expression of Mnfsh also began to increase in PL1. It is commonly know that, in the molting process, crustaceans experience morphological and appendage characteristic changes (Magalhães, 1989; Zhang et al., 2012). Therefore, our results indicate that the $M n f s h$ gene has a close relationship with metamorphosis of oriental river prawn.

In conclusion, our results indicate that Mnfsh may play important roles in embryogenesis, organogenesis, and morphological differentiation of $M$. nipponense. This study advances our understanding of the multiple biological functions of the Mnfsh gene. Additionally, our results enhance the understanding of the multiple biological functions of the Mnfsh genes and indicate that Mnfsh may play important roles in embryogenesis, organogenesis, and morphological differentiation of $M$. nipponense. However, the detailed molecular mechanism of the $M n f s h$ gene is still unknown in oriental river prawn; thus, its specific function needs to be further researched.

\section{ACKNOWLEDGMENTS}

Research supported by the National Science \& Technology Supporting Program of the 12th Five-year Plan of China (Grant \#2012BAD26B04), the Jiangsu Provincial Natural Science Foundation for Young Scholars of China (Grant \#BK2012091), the Science \& Technology Supporting Program of Jiangsu Province (Grant \#BE2012334), and the "Three New Projects" of Jiangsu Province (Grant \#D2013-6). 


\section{REFERENCES}

Amparyup P, Kondo H, Hirono I, Aoki T, et al. (2008). Molecular cloning, genomic organization and recombinant expression of a crustin-like antimicrobial peptide from black tiger shrimp Penaeus monodon. Mol. Immunol. 45: 1085-1093.

Bonasio R, Zhang G, Ye C, Mutti NS, et al. (2010). Genomic comparison of the ants Camponotus floridanus and Harpegnathos saltator. Science. 329: 1068-1071.

Budd GE and Telford MJ (2009). The origin and evolution of arthropods. Nature. 457: 812-817.

Bureau of Fishery, Ministry of Agriculture, P.R.C. (2011). Fisheries economic statistics. In: China Fishery Yearbook. China Agricultural Press, Beijing, p. 236.

Chen Y, Zhu Q, Chen H, Zhu XL, et al. (2012). The morphological and histological observation of embryonic development in the oriental river prawn Macrobrachium nipponense. J. Shanghai Ocean Univ. 21: 33-40.

Chintapalli VR, Wang J and Dow JA (2007). Using FlyAtlas to identify better Drosophila melanogaster models of human disease. Nat. Genet. 39: 715-720.

Digan ME, Haynes SR, Mozer BA, Dawid IB, et al. (1986). Genetic and molecular analysis of $f s(1) h$, a maternal effect homeotic gene in Drosophila. Dev. Biol. 114: 161-169.

Florence BL and Faller DV (2008). Drosophila female sterile (1) homeotic is a multifunctional transcriptional regulator that is modulated by Ras signaling. Dev. Dyn. 237: 554-564.

Forquignon F (1981). A maternal effect mutation leading to deficiencies of organs and homeotic transformations in the adults of Drosophila. Roux's Arch. Dev. Biol. 190: 132-138.

Gans M, Audit C and Masson M (1975). Isolation and characterization of sex-linked female-sterile mutants in Drosophila melanogaster. Genetics. 81: 683-704.

Gans M, Forquignon F and Masson M (1980). The role of dosage of the region 7d1-7d5-6 of the X chromosome in the production of homeotic transformations in Drosophila melanogaster. Genetics. 96: 887-902.

Haynes SR, Mozer BA, Bhatia-Dey N and Dawid IB (1989). The Drosophila fsh locus, a maternal effect homeotic gene, encodes apparent membrane proteins. Dev. Biol. 134: 246-257.

Haynes SR, Dollard C, Winston F, Beck S, et al. (1992). The bromodomain: a conserved sequence found in human, Drosophila and yeast proteins. Nucleic Acids Res. 20: 2603.

Hongtuo F, Sufei J and Yiwei X. (2012). Current status and prospects of farming the giant river prawn (Macrobrachium rosenbergii) and the oriental river prawn (Macrobrachium nipponense) in China. Aquac. Res. 43: 993-998.

Huang DH and Dawid IB (1990). The maternal-effect gene fsh is essential for the specification of the central region of the Drosophila embryo. New Biol. 2: 163-170.

Jeanmougin F, Wurtz JM, Le Douarin B, Chambon P, et al. (1997). The bromodomain revisited. Trends Biochem. Sci. 22: $151-153$.

Livak KJ and Schmittgen TD (2001). Analysis of relative gene expression data using real-time quantitative PCR and the $2^{(-\Delta \Delta C T)}$ method. Methods. 25: 402-408.

Lygerou Z, Conesa C, Lesage P, Swanson RN, et al. (1994). The yeast BDF1 gene encodes a transcription factor involved in the expression of a broad class of genes including snRNAs. Nucleic Acids Res. 22: 5332-5340.

Magalhães C (1989). The larval development of palaemonid shrimps from the Amazon Region reared in the laboratory. VI. Abbreviated development of Macrobrachium nattereri (Heller, 1862) (Crustacea: Decapoda). Amazoniana. 10: 379-392.

Marmorstein R and Berger SL (2001). Structure and function of bromodomains in chromatin-regulating complexes. Gene. 272: $1-9$.

Qiao H, Fu H, Jin S, Wu Y, et al. (2012). Constructing and random sequencing analysis of normalized cDNA library of testis tissue from oriental river prawn (Macrobrachium nipponense). Comp. Biochem. Physiol. Part D Genomics Proteomics. 7: 268-276.

Qiao H, Lv D, Jiang SF, Sun SM, et al. (2013). Genetic diversity analysis of oriental river prawn, Macrobrachium nipponense, in Yellow River using microsatellite marker. Genet. Mol. Res. 12: 5694.

Rhee K, Brunori M, Besset V, Trousdale R, et al. (1998). Expression and potential role of Fsrg1, a murine bromodomaincontaining homologue of the Drosophila gene female sterile homeotic. J. Cell Sci. 111:3541-3550.

Shearn A (1989). The ash-1, ash-2 and trithorax genes of Drosophila melanogaster are functionally related. Genetics. 121: 517-525.

Tamkun JW (1995). The role of brahma and related proteins in transcription and development. Curr. Opin. Genet. Dev. 5: 473-477.

Tamkun JW, Deuring R, Scott MP, Kissinger M, et al. (1992). Brahma: A regulator of Drosophila homeotic genes 
structurally related to the yeast transcriptional activator SNF2SWI2. Cell. 68: 561-572.

Tamura K, Peterson D, Peterson N, Stecher G, et al. (2011). MEGA5: molecular evolutionary genetics analysis using maximum likelihood, evolutionary distance, and maximum parsimony methods. Mol. Biol. Evol. 28: 2731-2739.

Thorpe KL, Abdulla S, Kaufman J, Trowsdale J, et al. (1996). Phylogeny and structure of the RING3 gene. Immunogenetics. 44: 391-396.

Zeng L and Zhou MM (2002). Bromodomain: an acetyl-lysine binding domain. FEBS Let. 513: 124-128.

Zhang F, Chen L, Wu P, Zhao W, et al. (2010). cDNA cloning and expression of Ubc9 in the developing embryo and ovary of oriental river prawn, Macrobrachium nipponense. Comp. Biochem. Physiol. B Biochem. Mol. Biol. 155: 288-293.

Zhang S, Jiang S, Xiong Y, Fu H, et al. (2014). Six chitinases from oriental river prawn Macrobrachium nipponense: cDNA characterization, classification and mRNA expression during post-embryonic development and moulting cycle. Comp. Biochem. Physiol. B. Biochem. Mol. Biol. 167: 30-40.

Zhang YP, Qiao H, Zhang WY, Sun SM, et al. (2012). Molecular cloning and expression analysis of two sex-lethal homolog genes during development in the oriental river prawn, Macrobrachium nipponense. Genet. Mol. Res. 12: 4698-4711.

Zhang Y, Fu H, Qiao H, Jin S, et al. (2013a). Molecular cloning and expression analysis of transformer-2 gene during development in Macrobrachium nipponense (de Haan 1849). J. World Aquac. Soc. 44: 338-349.

Zhang Y, Jiang S, Xiong Y, Sun S, et al. (2013b). Molecular cloning and expression analysis of extra sex combs gene during development in Macrobrachium nipponense. Turkish J. Fish. Aquat. Sci. 13: 331-340. 